\title{
Does upper-limb muscular demand differ between preferred and nonpreferred sitting pivot transfer directions in individuals with a spinal cord injury?
}

\author{
Dany Gagnon, PT, PhD; ${ }^{1-4}$ Alicia M. Koontz, PhD, RET; ${ }^{1,3 *}$ Eric Brindle; ${ }^{1,3}$ Michael L. Boninger, MD; ${ }^{1-3}$ \\ Rory A. Cooper, $\mathbf{P h D}^{\mathbf{1 - 3}}$ \\ ${ }^{1}$ Human Engineering Research Laboratories, Department of Veterans Affairs Pittsburgh Healthcare System, \\ Pittsburgh, PA; ${ }^{2}$ Departments of Physical Medicine and Rehabilitation, ${ }^{3}$ Rehabilitation Science and Technology, \\ and Bioengineering, University of Pittsburgh, Pittsburgh, PA; ${ }^{4}$ School of Rehabilitation, University of Montreal, \\ Montreal, Canada
}

\begin{abstract}
This study's main objective was to determine if upper-limb (UL) muscular demand was reduced when individuals with a spinal cord injury (SCI) performed a sitting pivot transfer (SPT) in the preferred direction compared with that in a nonpreferred direction. Fourteen individuals (mean +/standard deviation age $47.0+/-8.3 \mathrm{yr}$, height $1.80+/-0.08 \mathrm{~m}$, and weight $75.3+/-11.3 \mathrm{~kg}$ ) with SCI levels ranging from the sixth cervical to first sacral vertebra levels volunteered to participate in this study during the 2008 National Disabled Veterans Winter Sports Clinic. Surface electromyography (EMG) was used to record activity of the biceps, triceps, deltoid, pectoralis major, trapezius, and latissimus dorsi bilaterally during SPTs. These transfers were performed in each of the preferred and nonpreferred directions from the individuals' wheelchairs to a padded tub bench of even height. To quantify electromyographic muscular utilization ratio (MUR $\mathrm{EMG}_{\mathrm{G}}$ ), we normalized EMG data recorded during the transfer tasks to values obtained during static maximum voluntary contraction and then multiplied this ratio by 100 to obtain a percentage MUR $_{\text {EMG }}$ $\left(\% M U R_{E M G}\right)$. The overall peak \%MUR ${ }_{E M G}$ and the area under the \%MUR ${ }_{\mathrm{EMG}}$ curve were selected as primary outcome measures. Similar peak \%MUR ${ }_{\mathrm{EMGs}}$ were found between the preferred and nonpreferred transfer directions for all muscles from which data were recorded ( $p=0.053$ to 0.961 ). The peak $\% \mathrm{MUR}_{\mathrm{EMGs}}$ were also found to be similar between the leading and trailing ULs during the transfers in all muscles from which data were recorded ( $p=0.125$ to 0.838 ), except for the anterior deltoid, which was found to be solicited the most in the trailing $\mathrm{UL}(p=0.008)$. Comparable areas under the
\end{abstract}

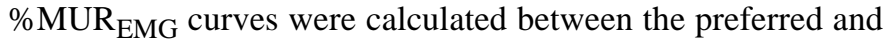
nonpreferred transfer directions for all muscles ( $p=0.289$ to 0.678 ) and between the leading and trailing ULs ( $p=0.104$ to 0.946). These results indicate that direction preference expressed by individuals with SCI when transferring between seats of even height is not explained by relative muscular demand differences.

Key words: activities of daily living, elbow, electromyography, paraplegia, rehabilitation, shoulder, spinal cord injury, task analysis, task performance, tetraplegia, upper limb.

\footnotetext{
Abbreviations: ANOVA = analysis of variance; $\mathrm{C}=$ cervical; $\mathrm{EMG}=$ electromyography, electromyographic; $\mathrm{EMG}_{\max }=$ maximum EMG value, $\mathrm{EMG}_{\text {transfer }}=\mathrm{EMG}$ data collected during transfers; HERL = Human Engineering Research Laboratories; $\mathrm{MUR}_{\mathrm{EMG}}=\mathrm{EMG}$ muscular utilization ratio; \%MUR $\mathrm{EMG}=$ percentage $\mathrm{MUR}_{\mathrm{EMG}} ; \mathrm{MVC}=$ maximal voluntary contraction; SCI = spinal cord injury; SD = standard deviation; sEMG = surface EMG; SPT = sitting pivot transfer; UL = upper limb; VA = Department of Veterans Affairs; WUSPI = Wheelchair User's Shoulder Pain Index.

* Address all correspondence to Dr. Alicia Koontz, HERL, VA Pittsburgh Healthcare System, 7180 Highland Dr, 151R1-H, Pittsburgh, PA 15206; 412-954-5287; fax: 412954-5340. Email: akoontz@pitt.edu DOI:10.1682/JRRD.2009.02.0012
} 


\section{INTRODUCTION}

Many individuals who sustain a spinal cord injury (SCI) will become long-term wheelchair users and will, consequently, typically perform sitting pivot transfers (SPTs) independently in their daily lives [1-2]. When performing SPTs using a side approach, individuals with SCI generally place one hand (leading hand) far enough away on or near the target surface, with the shoulder flexed and horizontally adducted, to allow displacement of the body weight and to leave adequate space for the buttocks to land at the end of the transfer [3]. Simultaneously, they normally place the other hand (trailing hand) beside the proximal half of the thigh slightly in front of the hip joint on or near the initial surface before performing the transfer [3]. From this starting position, they habitually flex and rotate their trunk and head forward and sideways while lifting their body weight off the initial surface and pivoting their buttocks in one twisting motion to the target surface [3]. The SPT terminates when the buttocks land beside the leading hand on the target surface and seated postural stability has been restored [3]. While doing so, individuals with SCI often express an SPT direction preference, though they are generally proficient in both directions.

Typical examples of SPTs include transferring from a wheelchair to a regular bed, tub/shower bench, toilet seat, treatment table, car seat, and vice versa. SPTs result in high mechanical loading (intensity) at the upper limbs (ULs) among individuals with SCI [4] since they are performed up to 35 times daily (frequency) [5]. Unsurprisingly, SPT may augment a person's likelihood of developing secondary impairments affecting the integrity of the skeletal, muscular, neurological, or vascular structures of the shoulder, elbow, or wrist joints over time [6]. Yet, compared with manual wheelchair propulsion or weight-relief lifts, SPT has not been studied extensively among long-term wheelchair users [6]. Additionally, no studies have ever attempted to explain why individuals with SCI generally prefer to perform SPTs in one direction compared with the other one.

Only a few biomechanical studies have specifically investigated SPTs over the past decade, and even fewer have attempted to quantify UL muscular demand during the completion of this task. Perry et al. report on the assessment of relative shoulder muscle activity using an electromyographic muscular utilization ratio $\left(\mathrm{MUR}_{\mathrm{EMG}}\right)$ approach to quantify muscular demand during SPTs among individuals with SCI [7]. They have confirmed that participants with complete paralysis of the abdominal muscles (level of SCI = eighth thoracic to first lumber) reached the most elevated mean MURs $_{\text {EMG }}$, expressed as a percentage of the maximum isometric manual muscle test (\%MUR $\mathrm{EMG}_{\mathrm{G}}$ ), during the lift phase of the transfer for the majority of muscles studied [7]. At this time, moderate (25-50 \%MUR $\mathrm{EMG}_{\text {) }}$ to high ( $>50 \% \mathrm{MUR}_{\mathrm{EMG}}$ ) values were recorded at the serratus anterior, latissimus dorsi, supraspinatus, infraspinatus, pectoralis major, anterior deltoid, and long head of the biceps [7]. Low activity ( $<25 \% \mathrm{MUR}_{\text {EMG }}$ ) was reported for the triceps during the SPTs. Similar mean \%MURs ${ }_{\text {EMG }}$ were found between the leading and trailing ULs during the lift phase of transfers for all the muscles investigated [7].

Finley et al. [8] also examined relative EMG activity during SPTs among long-term manual wheelchair users with and without shoulder impingement by using a normalization technique similar to the one Perry et al. used [7]. These two groups of participants demonstrated compara-

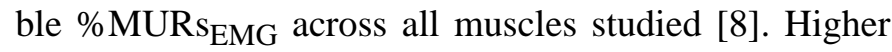
peak \%MURs ${ }_{\text {EMG }}$ were reported at the trailing UL than at the leading one for the anterior deltoid, serratus anterior, and lower trapezius at specific ranges of humeral elevation angles (second rotation), calculated within the plane of elevation (first rotation) [8]. However, cautious interpretation of these results is indicated because they only compared peak \%MUR ${ }_{E M G}$ values with specific humeral elevation angle ranges instead of comparing participants across specific phases of the transfer. For instance, the leading and trailing ULs may possibly have different roles within a similar humeral elevation range of movement or a specific humeral elevation range may be observed at different times during the transfers. Although these results may appear to disagree with those reported by Perry et al. [7], the two may be complementary because the peak \%MURs ${ }_{\mathrm{EMG}}$ may be more elevated in one UL than the other, whereas their mean values may remain equivalent.

Gagnon et al. investigated the effects of modifying target seat heights on the UL muscular demand during SPTs among individuals with SCI [9]. These results indicate that when individuals transferred to a target seat higher than the initial surface, greater mean EMG values were generated at the biceps of the leading UL as well as the deltoid and pectoralis major of the trailing UL than when they transferred toward a target seat of the same 
height [9]. However, lowering the target seat height with respect to the initial seat had no favorable effect on muscular demand [9].

The main objective of this study was to determine whether a UL muscular advantage (reduced relative muscular demand) exists when individuals perform SPTs in the preferred directions compared with doing so in the nonpreferred directions. The secondary objective of this study was to assess if differences in relative muscular demand existed between the leading and trailing ULs during SPT performance in either direction. To explain transfer direction preference, we hypothesized that the muscular demand in the preferred transfer direction would be less than that in the nonpreferred transfer direction. We also expected muscular demand to be higher in the trailing UL than in the leading UL during the SPTs.

\section{METHODS}

\section{Participants}

Fourteen individuals (13 males, 1 female) with an SCI volunteered to participate in this study during the 2008 National Disabled Veterans Winter Sports Clinic (Snowmass Village, Colorado). This clinic provides an opportunity for all veterans living with a disability to develop winter sports skills and to participate in a variety of workshops and educational sessions that will positively affect their rehabilitative journey and readjustment in their communities. On average (values throughout article are shown as mean \pm 1 standard deviation [SD] unless otherwise specified), participants were aged $47.0 \pm 8.3 \mathrm{yr}$, measured $1.80 \pm 0.08 \mathrm{~m}$ in height, weighed $75.3 \pm 11.3 \mathrm{~kg}$, and had sustained a complete $(n=4)$ or incomplete $(n=$ 10) SCI (levels of SCI varying between sixth cervical [C] and first sacral vertebrae) about $12.4 \pm 7.5 \mathrm{yr}$ before the study (minimum of $1 \mathrm{yr}$ ). The majority of participants were right-hand dominant (12/14). Individuals were eligible to participate in this study if they used a wheelchair as their primary source of mobility ( $>4$ hours a day in wheelchair) and if they had the ability to complete SPTs without human assistance. At the time of testing, no participants complained of any sign or symptom of trunk or UL secondary impairment that would have affected the way in which they routinely performed SPTs. The Wheelchair User's Shoulder Pain Index (WUSPI) scores ranged between 0 and $45(12.2 \pm 15.5$, median $=5)$ out of a maximum possible score of 150 , whereas the scores of the
WUSPI item specifically related to SPT (bed to wheelchair) ranged between 0 and $4(0.57 \pm 1.09$, median $=0)$ out of a maximum possible score of 10 for all participants [10]. This finding confirmed that shoulder pain had limited to no impact on their activities of daily living and on the performance of SPTs (tasks proposed in the current study). The study was completed at a temporary satellite laboratory space and managed, equipped, and supplied by the Human Engineering Research Laboratories (HERL) team during the 2008 National Disabled Veterans Winter Sports Clinic. Ethical approval was obtained from the Department of Veterans Affairs (VA) Pittsburgh Healthcare System Internal Review Board (02545). All participants were fully informed of the nature of the experimental protocol and signed an informed consent form before the study was initiated.

\section{Instrumentation}

We collected bilateral surface EMG (sEMG) for the sternal and clavicular fibers of the pectoralis major, anterior fibers of the deltoid, long head of the biceps and triceps brachii, inferior fibers of the trapezius, and the latissimus dorsi (14 channels) during the transfer tasks by using a 16-channel Noraxon Telemyo 2400T system (Noraxon USA Inc; Scottsdale, Arizona). We placed small disposable disc Ag-AgCl (silver/silver-chloride) surface electrodes (Ambu Inc; Blue Sensor Electrodes, N-00-S; Glen Bernie, Maryland) directly over the muscle belly of each muscle investigated parallel to the orientation of the muscle fibers using a bipolar configuration $(2 \mathrm{~cm}$ interelectrode distance). A description of the electrode placement for each muscle is presented in the Table. A reference electrode was placed at the center of the manubrium sternae (bony prominence). We fixed all EMG preamplified electrodes and electrode leads (length $=122 \mathrm{~cm}$ ) to the UL and trunk segments to minimize artifacts before being connected to one of the two 8-channel analog input Noraxon transmitting units (weight $=0.453 \mathrm{~kg}$ ) secured to the low back region with a belt positioned around the waist of each participant. We digitized all sEMG signals at a sampling frequency of $1,500 \mathrm{~Hz}$ before they were relayed to a portable computer using the MyoResearch XP Master Edition (Noraxon USA Inc) software to view, capture, and store the EMG data. An accelerator attached over the C7 spinous process (one channel) and four "in series" pressure sensors fixed underneath the buttocks (one channel) were connected to the Noraxon Telemyo 2400T system. Finally, a digital high-definition Webcam 
JRRD, Volume 46, Number 9, 2009

Table.

Schematic representation of experimental setup.

\begin{tabular}{lc}
\hline \multicolumn{1}{c}{ Muscle } & Placement of Electrodes \\
\hline Pectoralis Major (Clavicular Fibers) & $\begin{array}{c}\text { Midway between coracoid process and sternoclavicular joint } \\
2 \mathrm{~cm} \text { below clavicle. }\end{array}$ \\
Pectoralis Major (Sternal Fibers) & $\begin{array}{c}\mathrm{cm} \text { medial to anterior auxillary fold in direct vertical line with } \\
\text { coracoid process. }\end{array}$ \\
Deltoid (Anterior Fibers) & $2 \mathrm{~cm}$ distal and anterior to acromion. \\
Biceps Brachii (Long Head) & Center of muscle belly. \\
Triceps Brachii (Long Head) & $\begin{array}{c}\text { Midway between olecranon and acromion at } 2 \text { cm medial to line } \\
\text { over muscle belly. }\end{array}$ \\
Trapezius (Lower Fibers) & At two-thirds on line from trigonum spinae to 8th thoracic level \\
& spinous process. \\
Latissimus Dorsi & $2 \mathrm{~cm}$ inferior and lateral to inferior angle of scapula. \\
\hline \hline
\end{tabular}

(Microsoft ${ }^{\circledR}$ LifeCam VX-3000; San Jose, California) was linked by way of a USB (Universal Serial Bus) port to the MyoResearch XP Master Edition software to videotape all SPTs at $30 \mathrm{~Hz}$. We time-synchronized all signals collected and videotapes recorded to facilitate data analysis. Note that sEMG only allows monitoring of the UL superficial muscles and may not represent the EMG activity of the muscles lying underneath those studied.

\section{Maximum Voluntary Contractions}

Before realizing the SPT tasks, we performed static maximal voluntary contractions (MVCs) randomly using manual muscle testing according to a standardized protocol for all muscles investigated [11]. While remaining seated in their manual wheelchairs, participants were instructed to progressively increase their muscular effort to their maximal strength-generating capability while the evaluator manually resisted the movement with one hand and stabilized the proximal body segments with the other hand. The MVCs were maintained for a minimum of $3 \mathrm{~s}$ and a maximum of $7 \mathrm{~s}$. One MVC was recorded for each muscle investigated and a $30 \mathrm{~s}$ rest period separated each MVC for minimizing the development of fatigue. If a researcher or participant judged that the MVC was not reached, an additional MVC test was performed. Given the limits of this manual muscle testing method [12-13], these MVCs only provided an estimate of maximum EMG value $\left(E M G_{\max }\right)$ of each muscle required to normalize all EMG data collected during each transfer $\left(\mathrm{EMG}_{\text {transfer }}\right)$ and to quantify relative muscular demand (\%MUR $\mathrm{EMG}$ ) (see the "Outcome Measures" section).

\section{Transfer Tasks}

Participants were instructed to transfer from their personal wheelchair to a target height-adjustable padded tub bench and back (Newell Rubbermaid; Oakbrook, Illinois) set at a height equivalent to the combined height of each participant's wheelchair and seat cushion. During these transfers, participants were free to self-select their hand, feet, and buttocks positions to preserve their natural method of transfer. They positioned their trailing hands on their personal wheelchair (e.g., seat frame or wheel), slightly anterior to the hip joint, whereas their leading hand was positioned on the target bench far enough away to leave adequate space for the buttocks at the end of the transfer (Figure 1). After a familiarization period, we instructed participants to complete three transfers from the side in which they were most comfortable to determine preferred side measurements (task 1: preferred direction) and then to perform three transfers from the opposite side to determine the nonpreferred measurements (task 2: nonpreferred direction). Data were only recorded when participants were transferring from their own wheelchair to the padded tub bench in each of these two directions. We marked the initial positioning of the wheelchair (angle of wheelchair to bench), as well as the hand, feet, and buttocks positions, to assure similar starting positions across all trials and between the preferred and nonpreferred transfer directions for each participant. Participants were encouraged to use their regular movement strategies during transfers, especially in terms of amplitude and velocity. Although participants performed the SPTs in a laboratory environment, real-life situations were replicated as closely as possible when they performed 


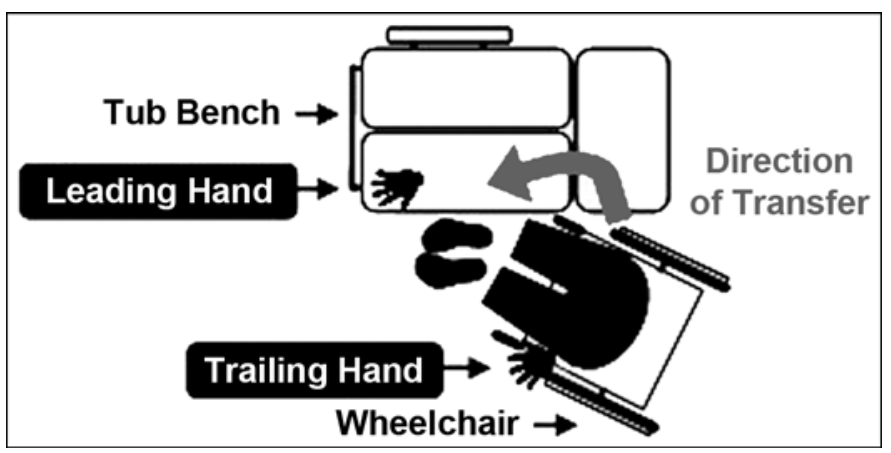

Figure 1.

Experimental setup of a sitting pivot transfer of individual with spinal cord injury.

the tasks. Rest periods were also given as needed to limit the effects of fatigue.

For each transfer, the start event marker corresponded to the start of the acceleration phase of the head and uppertrunk segments (C7) while the finish event marker of the transfer coincided with the end of the deceleration phase of these same segments. We further validated the position of the start and finish event markers by visually inspecting frame-by-frame the synchronized video sequence corresponding to each experimental trial. Note that the transfers were not time-normalized given the outcome measures described in the next section.

\section{Outcome Measures}

We visually inspected all EMG data collected following baseline noise removal (EMG signal recorded at rest) and thereafter filtered the data using a 4th-order Butterworth bandpass filter with cutoff frequencies set at 30 and $500 \mathrm{~Hz}$. The $30 \mathrm{~Hz}$ cutoff was expected to remove any possible electrocardiographic contaminations and artifacts [14]. Next, we full-wave rectified the EMG patterns before filtering them with a $6 \mathrm{~Hz}$ low-pass filter to finally generate linear envelopes. All EMG signal processing was performed digitally with a custom MATLAB program (The MathWorks; Natick, Massachusetts).

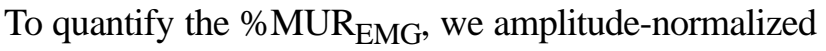
the $E M G_{\text {transfer }}$ against the $E M G_{\max }$ reached for each muscle investigated during the MVC and then multiplied this ratio by 100 to obtain a percentage: $\% \mathrm{MUR}_{\mathrm{EMG}}=$ $\left(\mathrm{EMG}_{\text {transfer }} / \mathrm{EMG}_{\text {max }}\right) \times 100$ [15-16]. We determined $\mathrm{EMG}_{\text {max }}$ of each muscle by finding the highest mean EMG values reached over consecutive periods of $0.5 \mathrm{~s}$ intervals during the MVC recorded, using a custom MATLAB program.

For each muscle investigated in this study, the peak ${ } \mathrm{MUR}_{\mathrm{EMG}}$ and the area under the \%MUR $\mathrm{EMG}_{\text {curve }}$ were selected as primary outcome measures. The peak $\% \mathrm{MUR}_{\mathrm{EMG}}$ value of each muscle was extracted between the start and end of the transfers. The \%MUR ${ }_{\mathrm{EMG}}$ was considered high if the value obtained exceeded 50 percent, moderate if in the range of 25 to 50 percent, and low if $<25$ percent [7]. We calculated the area under the $\% \mathrm{MUR}_{\mathrm{EMG}}$ curve, which can be considered as an overall indicator of muscular work as previously done in gait studies [17], by integrating the \%MUR $\mathrm{EMG}_{\mathrm{M}}$ curve between the start and end of the transfer. The amount of relative muscular work done by a muscle during the transfer tasks is proportional to the area measured under the $\mathrm{MUR}_{\mathrm{EMG}}$ curve [17]. We calculated these outcome measures using a custom MATLAB program for each trial and then for the statistical analyses computed and used a mean value of the three recorded trials in each direction.

\section{Statistics}

The normality of the distribution for the two outcomes of interest of each muscle investigated was first verified in each transfer direction with a one-sample Kolmogorov-Smirnov test. Since the normality of the distribution was confirmed for all peak \%MURs ${ }_{\text {EMG }}(p=$ $0.201-0.999)$ and areas under \%MUR $\mathrm{EMG}_{\text {curve values }}$ ( $p=0.087-0.995)$, we applied repeated measures analyses of variance (ANOVA) (two directions [preferred vs nonpreferred] $\times$ two UL roles [leading vs trailing]) separately for each muscle investigated to determine if the transfer direction or UL roles played a part in the muscular demand measured during transfers. Since no interaction effects were found when ANOVAs were conducted, only main effects were reported separately for each muscle investigated. A level of significance of $p<0.05$ was selected for all tests. Statistical analyses were performed with SPSS ${ }^{\circledR}$ v14.0 software (Chicago, Illinois) for Windows.

\section{RESULTS}

All participants were able to complete all transfers independently from their preferred sides as well as from their nonpreferred sides. To do so, participants positioned their own wheelchair (initial seat) at an angle varying between $8^{\circ}$ and $50^{\circ}\left(22.4^{\circ} \pm 12.2^{\circ}\right)$ with respect to the 
tub bench (target seat). They also required between 0.48 and $3.58 \mathrm{~s}(2.25 \pm 0.68 \mathrm{~s})$ to perform the transfer tasks investigated (total time between start and finish event markers). When transferring in the preferred direction, half the participants $(n=7)$ used their dominant UL as the trailing one, whereas the other half $(n=7)$ used their dominant UL as the leading one.

For all muscles investigated, the overall peak $\% \mathrm{MUR}_{\mathrm{EMG}}$ (group mean $\pm 1 \mathrm{SD}$ ) and the area under the

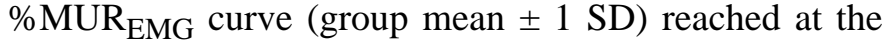
leading and trailing ULs when participants transferred in the preferred and nonpreferred directions are shown in Figures 2 and 3, respectively. Similar peak \%MURs ${ }_{\text {EMG }}$ were found between the preferred and nonpreferred transfer directions for all muscles ( $p=0.053-0.961)$. The

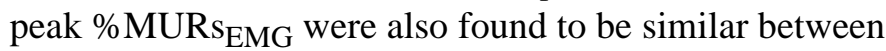
the leading and trailing ULs during the transfers in almost all muscles ( $p=0.125-0.838$ ). Only the peak $\%$ MURs $_{\text {EMG }}$ of the anterior fibers of the deltoid were found to differ significantly between the leading and trailing ULs ( $p=0.008)$, independently of the transfer direction, with the trailing UL having $>25.1$ percent peak muscle demand than the leading one. Similar areas under the $\% \mathrm{MUR}_{\mathrm{EMG}}$ curves were calculated between the preferred and nonpreferred transfer directions for all muscles ( $p=0.289-0.678)$ and between the leading and trailing ULs $(p=0.104-0.946)$.

\section{DISCUSSION}

\section{Preferred Versus Nonpreferred Directions}

The results of this study do not support the hypothesis that the muscular demand is greater during SPTs performed in the nonpreferred direction than SPTs performed in the preferred direction. In fact, transferring in the nonpreferred and the preferred directions was found to require similar muscular demand (peak $\% \mathrm{MUR}_{\mathrm{EMG}}$ values and the areas under the $\% \mathrm{MUR}_{\mathrm{EMG}}$ curves) for all muscles investigated. This finding may be explained by the fact that long-term manual wheelchair users with SCI generally perform SPTs in both directions (preferred and nonpreferred) numerous times daily while minimizing distance and height difference between seats as much as possible. This action may help develop (early motor stage) and refine (motor consolidation) comparable rapid and coordinated motor performance for transferring in both directions [18]. Overtime, it may also lead to developing and consolidating an internal model [19]. (a) 1
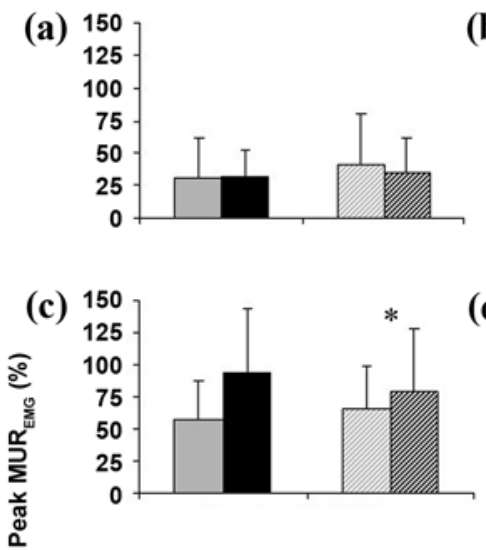

(e)

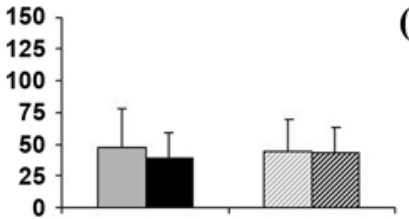

(g)

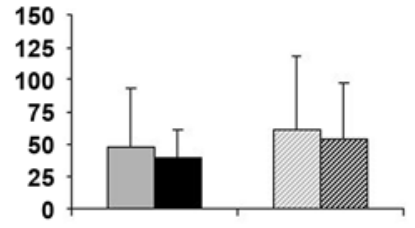

(b)

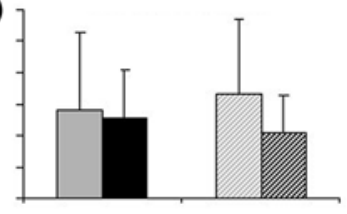

(d)

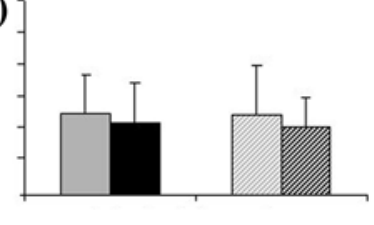

(f)

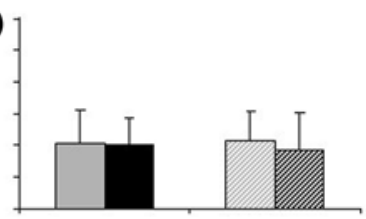

Figure 2.

Group mean \pm 1 standard deviation peak percentage electromyographic muscular utilization ratio (MUR $\mathrm{EMG}_{\mathrm{EM}}$ ) of muscles investigated at leading and trailing upper limbs (ULs) when persons with spinal cord injury transferred in preferred and nonpreferred directions: (a) biceps brachii, (b) triceps brachii, (c) anterior deltoid, (d) pectoralis major (sternal fibers), (e) pectoralis major (clavicular fibers), (f) inferior trapezius, and (g) latissimus dorsi. * Main effect for UL role; $p=0.008$.

Such a model may allow the central nervous system to adequately preplan and calibrate the force production (muscle activity) required for an individual with SCI to perform SPTs in both directions, even before motiondependent feedback becomes available, suggesting a feed-forward control strategy [19]. This last point is further supported by the fact that the role the dominant UL played spontaneously varied equally (leading vs trailing) across participants when transferring in the preferred direction. Transfer direction preference may plausibly become more relevant if the complexity level of the sitting transfer tasks is gradually progressed (i.e., target seat raised with respect to the initial seat, presence of a gap between the initial and target seats, or both) or if participants are unfamiliar with the experimental tasks (i.e., 


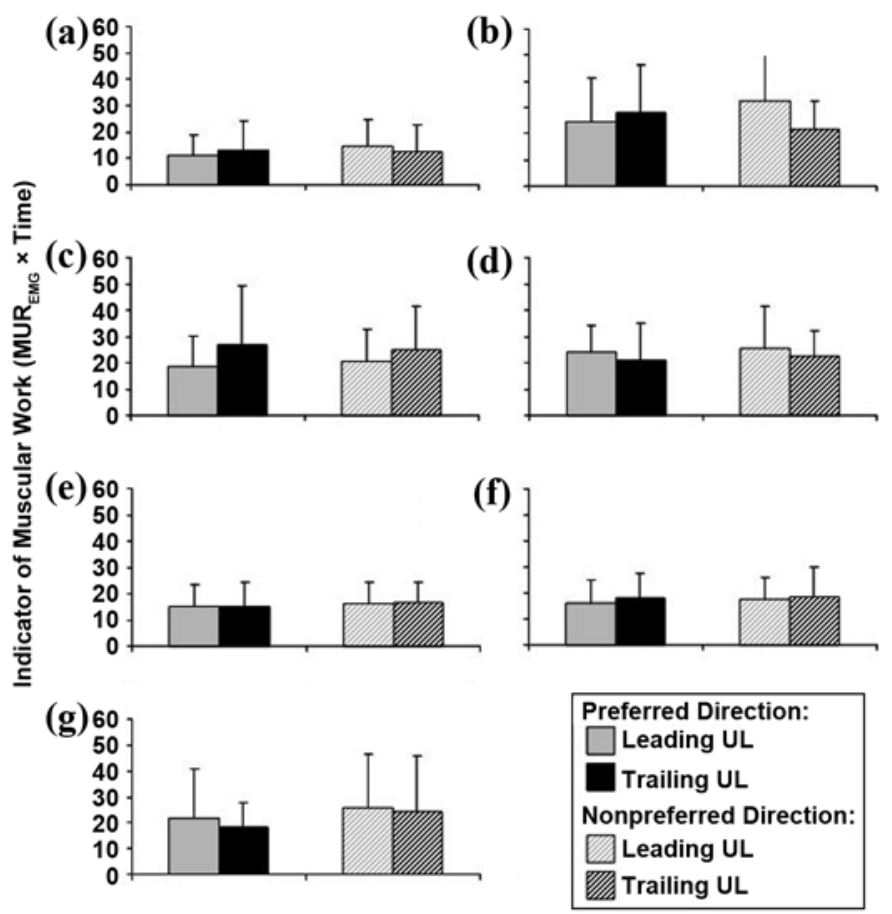

Figure 3.

Group mean \pm 1 standard deviation area under percentage electromyographic muscular utilization ratio (MUR $\mathrm{EMG}_{\mathrm{E}}$ ) curve of muscles investigated (indicator of muscular work) at leading and trailing upper limbs (ULs) when transferring in preferred and nonpreferred directions: (a) biceps brachii, (b) triceps brachii, (c) anterior deltoid, (d) pectoralis major (sternal fibers), (e) pectoralis major (clavicular fibers), (f) inferior trapezius, and (g) latissimus dorsi.

transferring to an unstable target seat $15 \mathrm{~cm}$ higher and $15 \mathrm{~cm}$ farther than the initial seat). Given the context of realization of the current study, we investigated the SPT tasks that individuals with an SCI most commonly performed in daily life and those they most likely safely completed (high familiarity/low complexity). Caution is therefore warranted when extrapolating these results to other transfer tasks.

\section{Leading Versus Trailing Upper Limbs}

The results of this study weakly support the hypothesis that the muscular demand is higher in the trailing UL than in the leading UL during the SPTs, independent of the direction of preference. Only the anterior fibers of the deltoid muscles, which predominantly act as an abductor and flexor of the shoulder (glenohumeral joint), reached a significantly higher peak muscular demand in the trailing UL than in the leading UL during the SPTs, independently of their directions. This finding partially corroborates the findings of Finley et al., who documented higher peak ${ }_{\% M U R}{ }_{E M G}$ at the trailing UL than at the leading one for the anterior deltoid, serratus anterior, and lower trapezius at specific ranges of humeral elevation angles during

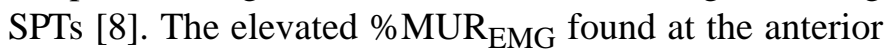
deltoid may be explained, in part, by the fact that the trailing UL progresses into abduction and flexion during the completion of the transfers (longer lever arm) [3,20]. Consequently, the shoulder generating substantial moments (net eccentric adduction and concentric flexion moments) becomes critical to simultaneously support body weight, push body weight away from the initial seat (i.e., wheelchair), and prevent a fall $[4,20]$. Moreover, the trailing UL is also known to counteract greater vertical and horizontal reaction forces than the leading UL early during SPTs, more precisely around the time the lift phase is initiated [5,21]. Despite peak EMG differences being reported, previous work has confirmed similar

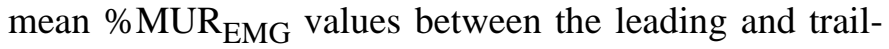
ing ULs during the lift phase of the transfer for all muscles investigated, including the anterior deltoid [7]. These mean $\%$ MUR $_{\text {EMG }}$ values may not represent the peak $\% \mathrm{MUR}_{\mathrm{EMG}}$ intensity. Yet, they may support our findings also, confirming similar overall relative muscular work (area under the MUR $\mathrm{EMG}_{\mathrm{E}}$ curve) found between the leading and trailing ULs for all muscles investigated.

As for the leading UL, previous studies have reported that it is predominantly used to simultaneously support body weight, move body mass toward the target seat (i.e., tub bench), and prevent a fall $[3,20]$. At this shoulder, these combined actions translate into elevated net concentric shoulder flexor and adductor moments being generated during SPTs [4,20]. For the adduction moments being generated at the shoulders, less EMG activity is needed during eccentric contraction (trailing shoulder) than during concentric contraction (leading shoulder) [22]. Unsurprisingly, the highest peak \%MURs ${ }_{\mathrm{EMG}}$, though not statistically significant, were generally observed in the leading UL.

\section{Clinical Relevance}

Based on the results of the current study, clinicians should continue to assess and train SPTs in both directions (preferred and nonpreferred) during rehabilitation, specifically when no UL strength asymmetry or UL secondary impairment is present. Task-specific training can potentially translate into the development and refinement of rapid and coordinated motor performance needed for 
transferring in both directions. Transfer direction preference cannot always be accommodated in the natural environment. The results of the current study also validate that SPTs can be ranked as one of the most demanding wheelchair-related activities routinely performed by individuals with SCI, as previously suggested [6,23-25].

Since the results of the current study highlight moderate to high muscular demand in the majority of the muscles investigated, clinicians should not neglect to assess and retrain muscle strength, especially at the shoulder. Given the frequency and intensity (cumulative trauma disorder), increasing "absolute" muscle strength at the ULs may plausibly diminish a person's likelihood of developing secondary musculoskeletal impairments (decrease "relative" muscular demand [MUR]) affecting the ULs over time. In the same way, the use of human assistance or a technical aid (e.g., sliding board) when individuals perform SPTs during rehabilitation may appear desirable until sufficient strength-generating and coordination capability have been reached. Finally, confirming a general rule for the role of a specific UL during SPTs would seem premature based on the isolated differences found in the present study between the leading and trailing anterior fibers of the deltoid.

\section{Limitations}

The results of this study should be interpreted carefully for several reasons. First, the number of participants is limited. Nonetheless, the current sample size is comparable with those commonly used in other EMG studies investigating functional activities in individuals with an SCI. The current results will facilitate sample size calculation in future studies aiming to increase the strength of the current evidence. The fact that only veterans participating in the 2008 National Disabled Veterans Winter Sports Clinic were included in this study also needs to be considered (potential population bias). Second, the severity of the sensory-motor impairments, which primarily relates to the level and degree of completeness of the lesion sustained to the spinal cord, varied widely across participants. For example, three participants had an incomplete lesion to the spinal cord and the ability to support part of their body weight through their lower limbs. Although these participants may have been able to reduce the amount of weight supported by their ULs, its reduction most likely had no effect on the transfer direction preference because their lower limbs were expected to support a similar amount of body weight in each trans- fer direction. For these participants, we acknowledge that their MURs may have been reduced to some extent, but no effects were anticipated on the outcomes of this study (statistics) because they were compared with themselves across all tasks.

Finally, these results should be interpreted carefully because similar \%MURs ${ }_{\mathrm{EMG}}$ (relative values) do not necessarily confirm symmetrical strength-generating capability in the UL muscle investigated. Despite the presence of similar MUR between the transfer in the nonpreferred direction and that performed in the preferred direction, the presence of strength-generating capability asymmetry remains possible and may still explain, to some extent, transfer direction preference. Future studies focusing on SPTs should measure bilateral UL strength and quantify other factors possibly associated with transfer direction preference (i.e., lower-limb spasticity, seated postural stability, shoulder proprioception, UL perception of effort). One also needs to consider that similar $\mathrm{MUR}_{\mathrm{EMG}}$ (relative value) may also hide different movement strategies and levels of muscular cocontraction during the performance of SPTs. For example, the large SDs observed at the triceps and anterior deltoid could be linked to the magnitude of participants' forward trunk inclination used during the task because forward trunk inclination modifies the orientation of the reaction force vector underneath hands and the lever arm (perpendicular distance from vector to joint axis of rotation) (Figure 4). Mechanically, an elbow

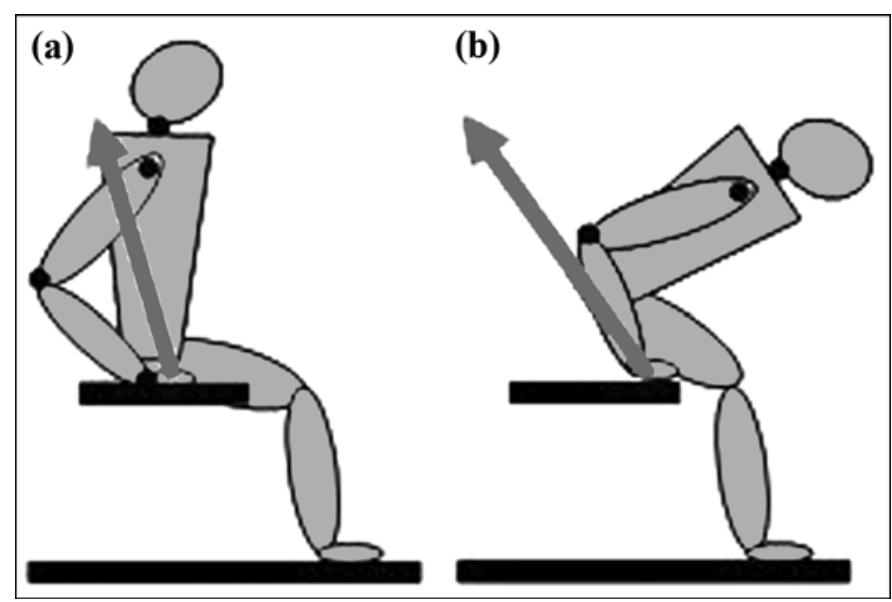

Figure 4.

Effects of movement strategy, particularly of magnitude of forward trunk inclination, on orientation of reaction force recorded underneath hands during sitting pivot transfers: (a) upright axial skeleton and (b) forward trunk inclination strategies. 
extension (triceps) or flexion (biceps) moment is needed to support body weight with the ULs during the lift phase of the transfer when the reaction force passes in front (Figure 4(a)) or behind (Figure 4(b)) the elbow joint axis, respectively, in a sagittal plane. Shoulder flexion moment (anterior deltoid, pectoralis major) is expected to progress when forward trunk inclination increases, since the lever arm is enlarged (Figure 4). Hence, different movement strategies or levels of muscular cocontraction may partly explain transfer direction preference. For this reason, the quantification of movement strategies (kinematics) and of net moments around the shoulder joints (kinetics), in complement to the UL muscle EMG assessment, could certainly help one better understand transfer direction preference.

\section{CONCLUSIONS}

The results of this study indicate that muscular demand is similar during SPTs performed in the nonpreferred direction and those performed in the preferred direction. They also help one better understand the muscular demand required to complete SPTs because the anterior fibers of the deltoid muscles were more solicited in the trailing UL than in the leading UL during the SPTs, independent of transfer direction preference. A more comprehensive methodology may be needed in future studies to reveal factors that explain transfer direction preference often expressed by individuals with SCI.

\section{ACKNOWLEDGMENTS}

\author{
Author Contributions: \\ Study concept and design: D. Gagnon, A. M. Koontz, M. L. Boninger, \\ R. A. Cooper. \\ Acquisition of data: D. Gagnon, A. M. Koontz, R. A. Cooper. \\ Analysis and interpretation of data: D. Gagnon, A. M. Koontz, \\ E. Brindle. \\ Drafting of manuscript: D. Gagnon, A. M. Koontz, E. Brindle. \\ Critical revision of manuscript for important intellectual content: \\ D. Gagnon, A. M. Koontz, M. L. Boninger, R. A. Cooper. \\ Statistical analysis: D. Gagnon, A. M. Koontz, E. Brindle. \\ Obtained funding: A. M. Koontz, R. A. Cooper. \\ Administrative, technical, or material support: D. Gagnon, \\ A. M. Koontz. \\ Study supervision: D. Gagnon, A. M. Koontz.
}

Financial Disclosures: The authors have declared that no competing interests exist.
Funding/Support: This material was based on work supported in part by the VA, Office of Research and Development, Rehabilitation Research and Development Service, grants A4489R and B3142C; the National Science Foundation, grant EEC 0552351; and a donation from the Paralyzed Veterans of America. This material is the result of work supported with resources and the use of facilities at HERL, VA Pittsburgh Healthcare System. Dr. Gagnon held a postdoctoral research scholarship from the Fond de la recherché en santé du Québec at the time of the study.

Additional Contributions: We gratefully acknowledge the contributions of Emily Teodorski and Rosemarie Cooper to this study.

Participant Follow-Up: The authors do not plan to inform participants of the publication of this study. However, participants have been encouraged to check the HERL Web site or consult the HERL

Quarterly Newsletter for updated publications.

\section{REFERENCES}

1. Allison GT. The ability to transfer in individuals with spinal cord injury. Crit Rev Phys Med Rehabil. 1997;9(2): 131-50. [PMID: 11114770] DOI:10.1038/sj.sc.3101070

2. Nyland J, Quigley P, Huang C, Lloyd J, Harrow J, Nelson A. Preserving transfer independence among individuals with spinal cord injury. Spinal Cord. 2000;38(11):649-57. [PMID: 11114770] DOI:10.1038/sj.sc.3101070

3. Gagnon D, Nadeau S, Noreau L, Eng JJ, Gravel D. Trunk and upper extremity kinematics during sitting pivot transfers performed by individuals with spinal cord injury. Clin Biomech (Bristol, Avon). 2008;23(3):279-90.

[PMID: 18037198] DOI:10.1016/j.clinbiomech.2007.09.017

4. Gagnon D, Nadeau S, Noreau L, Dehail P, Piotte F. Comparison of peak shoulder and elbow mechanical loads during weight-relief lifts and sitting pivot transfers among manual wheelchair users with spinal cord injury. J Rehabil Res Dev. 2008;45(6):863-73. [PMID: 19009472] DOI:10.1682/JRRD.2007.11.0189

5. Gagnon D, Nadeau S, Noreau L, Dehail P, Gravel D. Quantification of reaction forces during sitting pivot transfers performed by individuals with spinal cord injury. J Rehabil Med. 2008;40(6):468-76.

[PMID: 18509563] DOI:10.2340/16501977-0192

6. Consortium for Spinal Cord Medicine. Preservation of upper limb function following spinal cord injury: A clinical practice guideline for health-care professionals. Washington (DC): Consortium for Spinal Cord Medicine; 2005.

7. Perry J, Gronley JK, Newsam CJ, Reyes ML, Mulroy SJ. Electromyographic analysis of the shoulder muscles during depression transfers in subjects with low-level paraplegia. 
Arch Phys Med Rehabil. 1996;77(4):350-55.

[PMID: 8607758]

DOI:10.1016/S0003-9993(96)90083-0

8. Finley MA, McQuade KJ, Rodgers MM. Scapular kinematics during transfers in manual wheelchair users with and without shoulder impingement. Clin Biomech (Bristol, Avon). 2005;20(1):32-40. [PMID: 15567534] DOI:10.1016/j.clinbiomech.2004.06.011

9. Gagnon D, Nadeau S, Noreau L, Eng JJ, Gravel D. Upper extremity muscle activation patterns during sitting pivot transfers performed by individuals with complete spinal cord injury. J Electromyogr Kinesiol. 2009;19(3):509-20.

10. Curtis KA, Roach KE, Applegate EB, Amar T, Benbow CS, Genecco TD, Gualano J. Development of the Wheelchair User's Shoulder Pain Index (WUSPI). Paraplegia. 1995; 33(5):290-93. [PMID: 7630657]

11. Hislop HJ, Montgomery J, Connolly BH, Daniels L. Daniel's and Worthingham's muscle testing: Techniques of manual examination. Philadelphia (PA): W. B. Saunders; 2007. p. 61-177.

12. Bohannon RW, Corrigan D. A broad range of forces is encompassed by the maximum manual muscle test grade of five. Percept Mot Skills. 2000;90(3 Pt 1):747-50.

[PMID: 10883753]

DOI:10.2466/PMS.90.3.747-750

13. Knepler C, Bohannon RW. Subjectivity of forces associated with manual-muscle test grades of 3+, 4-, and 4. Percept Mot Skills. 1998;87(3 Pt 2):1123-28.

[PMID: 10052069]

14. Butler HL, Newell R, Hubley-Kozey CL, Kozey JW. The interpretation of abdominal wall muscle recruitment strategies change when the electrocardiogram (ECG) is removed from the electromyogram (EMG). J Electromyogr Kinesiol. 2009;19(2):102-13. [PMID: 18055221]

DOI:10.1016/j.jelekin.2007.10.004

15. Gagnon D, Nadeau S, Gravel D, Noreau L, Larivière C, Gagnon D. Biomechanical analysis of a posterior transfer maneuver on a level surface in individuals with high and low-level spinal cord injuries. Clin Biomech (Bristol, Avon). 2003;18(4):319-31. [PMID: 12689782] DOI:10.1016/S0268-0033(03)00016-0

16. Gagnon D, Nadeau S, Gravel D, Noreau L, Larivière C, McFadyen B. Movement patterns and muscular demands during posterior transfers toward an elevated surface in individuals with spinal cord injury. Spinal Cord. 2005; 43(2):74-84. [PMID: 15356677] DOI:10.1038/sj.sc.3101660
17. Milot MH, Nadeau S, Gravel D, Bourbonnais D. Effect of increases in plantarflexor and hip flexor muscle strength on the levels of effort during gait in individuals with hemiparesis. Clin Biomech (Bristol, Avon). 2008;23(4):415-23. [PMID: 18082922]

DOI:10.1016/j.clinbiomech.2007.11.003

18. Seidler RD, Noll DC, Thiers G. Feedforward and feedback processes in motor control. Neuroimage. 2004;22(4):1775-83. [PMID: 15275933]

DOI:10.1016/j.neuroimage.2004.05.003

19. Desmurget M, Grafton S. Forward modeling allows feedback control for fast reaching movements. Trends Cogn Sci. 2000;4(11):423-31. [PMID: 11058820] DOI:10.1016/S1364-6613(00)01537-0

20. Gagnon D, Nadeau S, Desjardins P, Noreau L. Biomechanical assessment of sitting pivot transfer tasks using a newlydeveloped instrumented transfer system among long-term wheelchair users. J Biomech. 2008;41(5):1104-10.

[PMID: 18191866]

DOI:10.1016/j.jbiomech.2007.11.021

21. Forslund EB, Granström A, Levi R, Westgren N, Hirschfeld $\mathrm{H}$. Transfer from table to wheelchair in men and women with spinal cord injury: Coordination of body movement and arm forces. Spinal Cord. 2006;45(1):41-48.

[PMID: 16670685]

DOI:10.1038/sj.sc.3101935

22. Lieber RL, Bodine-Fowler SC. Skeletal muscle mechanics: Implications for rehabilitation. Phys Ther. 1993;73(12): 844-56. [PMID: 8248293]

23. Van Drongelen S, Van der Woude LH, Janssen TW, Angenot EL, Chadwick EK, Veeger DH. Mechanical load on the upper extremity during wheelchair activities. Arch Phys Med Rehabil. 2005;86(6):1214-20. [PMID: 15954062] DOI:10.1016/j.apmr.2004.09.023

24. Van Drongelen S, Van der Woude LH, Janssen TW, Angenot EL, Chadwick EK, Veeger DH. Glenohumeral contact forces and muscle forces evaluated in wheelchairrelated activities of daily living in able-bodied subjects versus subjects with paraplegia and tetraplegia. Arch Phys Med Rehabil. 2005;86(7):1434-40. [PMID: 16003677] DOI:10.1016/j.apmr.2005.03.014

25. Bayley JC, Cochran TP, Sledge CB. The weight-bearing shoulder. The impingement syndrome in paraplegics. J Bone Joint Surg Am. 1987;69(5):676-78.

[PMID: 3597466]

Submitted for publication February 5, 2009. Accepted in revised form September 9, 2009. 